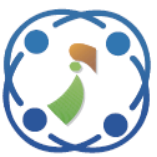

\title{
Deep Neural Glow Worm Swarm Optimized Soft C-Means Clustering for Energy Aware Route Discovery and Data Gathering in WSN
}

\author{
Sridhar Ramaiah ${ }^{1 *}$ \\ Guruprasad Nagraj ${ }^{2}$ \\ ${ }^{1}$ Department of Information Science and Engineering, \\ Global Academy of Technology, affiliated to VTU, Bengaluru \\ ${ }^{2}$ Department of Computer Science and Engineering, Global Academy of Technology, affiliated to VTU, Bengaluru \\ * Corresponding author's Email: srimln77@ gmail.com
}

\begin{abstract}
Cluster head selection enacts a prominent role in Wireless Sensor Network to optimize the energy usage during the data collection. Few research works have been designed to choose the best cluster head in wireless network using different optimization techniques. However, cluster head selection performance of conventional algorithms was lower to extend the lifetime of network. Therefore, a Deep Neural Glowworm Swarm Optimized Soft C-Means Clustering (DNGSOSCC) model is proposed. Initially, DNGSOSCC model obtains number of sensor nodes as input at the input layer. After taking input, soft clustering process is carried in DNGSOSCC model at the first hidden layer where it groups the sensor nodes in WSN into a different cluster. Then, the glow worm population is initialized in DNGSOSCC model with the support of number of clusters formed at the second hidden layer. Next, Luciferin value is assessed in DNGSOSCC model for all sensor nodes according to their residual energy level in the third hidden layer. Followed by, the sensor node with higher luciferin value within cluster is selected as optimal cluster head at the fourth hidden layer to perform energy efficient data gathering in WSN. Finally, the output layer provides the selection result of optimal cluster heads in WSN. By using the above process, DNGSOSCC model significantly gathers the data from its cluster member through selected optimal cluster heads with minimal amount of energy. From that, DNGSOSCC model upsurges the lifespan of network through performing efficient route discovery and data collection in WSN. The DNGSOSCC model conducts the simulation work using metrics such as data deliverance ratio, data loss ratio, data transmission delay and network lifetime with reverence to number of data packets and sensor nodes. From the experiments conducted, DNGSOSCC model improves the data deliverance ratio and network lifetime by $19 \%$ and $10 \%$ as well as minimizes the data loss ratio and data transmission delay by $76 \%$ and $34 \%$ respectively than existing methods.
\end{abstract}

Keywords: Cluster head, Data collection, Deep neural learning, Glow warm population, Luciferin value, Residual energy level, Soft c-means clustering.

\section{Introduction}

The major notion of WSN is to employ sensors to amass information around adjoining areas and convey it to base station. Sensors disseminated in a certain environment endure stationary, with power enduring a foremost problem. Many etiquettes and algorithms have been developed for energy efficient data gathering via optimal cluster head selection. However, the energy diminution during the data collection process was higher. Therefore, a novel optimal cluster head selection technique called DNGSOSCC standard is proposed in this article.

A fuzzy based balanced cost $\mathrm{CH}$ selection procedure (FBECS) was employed [1] to lug out data amassing in WSN with minimal energy. However, data transmission delay using FBECS was higher. An enhanced clustering hierarchy $(\mathrm{ECH})$ approach was employed in [2] for energy efficiency in WSNs. But, network lifetime was lower.

A novel routing and data gathering was performed in [3] with application of modified expectation-maximization. However, energy usage 
was higher. A Hybrid Artificial Bee Colony and Monarchy Butterfly Optimization Algorithm were combined in [4] to increase the cluster head selection performance. But, data loss rate was higher.

Firefly algorithm was employed [5] in order to improve the vivacity efficiency of network and lifetime of nodes through electing best cluster head with minimal distance. However, cluster head selection performance was not enough to get higher data delivery rate. Projection-based compressive data gathering (CDG) was performed [6] to collect data from dense sensor networks. But, energy utilization was remained open issue.

Fuzzy modelling based energy aware clustering was accomplished [7] to boost lifetime of network and to resolve the problem of energy preservation in sensor networks. However, data delivery rate was not higher. An enhanced Artificial Bee Colony Optimization-based Clustering Technique (IABCOCT) was presented [8] for carrying out optimal clustering process and selection of cluster heads. But, transmission delay was not reduced.

An energy-efficient adaptive overlapping clustering (EEAOC) technique was employed [9] to get longer network lifetime cycle in WSN. Conversely, better energy efficiency was not obtained. Fitness based Glow worm swarm through Fruit fly procedure (FGF) was introduced [10] to determine the best $\mathrm{CH}$ in WSN by considering energy and delay constraints. But, proportion of number of packets that are exactly reached at the destination was lower.

A secure and energy aware clustering was performed in [11] with help of reliable moth flame optimization and genetic algorithm. However, optimal cluster head selection was not considered. Genetic Algorithm-based Optimized Clustering (GAOC) protocol was employed in [12] for optimized $\mathrm{CH}$ selection using fitness function calculation. But, energy effective data gathering was not performed.

A Swarm Optimization-Enhanced Data Aggregation Tree Based on a Non uniform Clustering formation was developed in [13] to decrease the delay and energy diminution in WSN. However, network lifetime was lower. Energy aware competition based clustering was presented in [14] for choosing cluster head in WSN. But, delay during the transmission process was very high.

Bio-Inspired Based Approach was implemented in [15] to increase the node clustering performance in WSN and thereby minimizing the data loss. However, energy and network life time was not considered. A path based clustering was carried out in [16] with help of Ant Colony Optimization (ACO) to discover the finest cluster head with smallest routing path and vitality utilization. But, ratio of number of packets vanished during the routing process was high.

A sustainable data gathering technique was introduced in [17] with application of nature inspired optimization in WSNs to minimize the transmission cost and overhead of network. However, data loss was higher. A hybrid GGWSO (Grouped Grey Wolf Search Optimisation) procedure was applied [18] to obtain a cluster head selection performance in WSN. But, network lifespan was lower.

High-quality clustering ideal cluster head selection was performed [19] with the support of fuzzy logic to get better scalability performance. However, error rate during the clustering process was higher. Multi-scale Optimized Clustering was accomplished in [20] for optimizing energy in WSN. But, packet delivery ratio was lower.

To resolve the above said traditional issues, DNGSOSCC model is proposed in this paper. The main contributions of DNGSOSCC model is presented beneath,

- To upgrade the recital of energy effective data aggregation in WSN through cluster head when related to contemporary works, DNGSOSCC model is introduced in this work by combining glow worm swarm optimization and soft cmeans clustering and deep neural learning concepts.

- To enhance the selection performance of optimal cluster head for data collection compared to traditional mechanisms, the method of deep neural learning algorithm is applied in DNGSOSCC model with help of glow worm swarm optimization. Thus, DNGSOSCC model used behaviour of glow worms (i.e. fireflies) to find the cluster head with high enduring energy as best in wireless network for data aggregation.

The rest of paper structure is framed as follows. Section 2 illustrates an extensive process of proposed DNGSOSCC model using architectural schema. In Section 3, simulation setup is designated. Section 4 demonstrates the simulation outcomes of proposed DNGSOSCC model and compared with two existing methods. Lastly, Section 5 concludes the article.

\section{Deep neural glow worm swarm optimized soft c-means clustering model}

The Deep Neural Glow worm Swarm Optimized Soft C-Means Clustering (DNGSOSCC) model is developed to obtain energy effectual data gathering in WSN through optimal cluster head selection. The DNGSOSCC model is introduced in this research work with the application of Glow worm Swarm 
Optimization and Soft C-Means Clustering concepts in deep neural learning. The designed DNGSOSCC model is an artificial neural network with several layers amid the input and output layers. Besides, DNGSOSCC model is a feedforward linkage in which data streams from the input layer to the output layer without looping back. On the contrary to traditional works, deep neural learning concepts is utilized in DNGSOSCC model as it helps to handle the huge quantity of sensor nodes during the process of cluster formation.

Moreover, deep neural learning applied in DNGSOSCC model is an artificial intelligence function. This deep learning employs the workings of the human brain and has capable of learning sensor nodes behaviours in unstructured network. Also, Glow worm Swarm Optimization applied in DNGSOSCC model helps to provide the global optimal solution for cluster head selection problem. Besides, Soft C-Means Clustering in DNGSOSCC model supports to efficiently form different number of clusters in WSN with least time. The architectural outline of DNGSOSCC model is exhibited in Fig. 1. Fig. 1 exhibits the whole process of DNGSOSCC model to perform energy aware route discovery and data gathering in WSN. As presented in the above architecture representation, DNGSOSCC model comprises of three layers precisely input, hidden and output layer. Let us consider a WSN with enormous number of sensor nodes. Initially, input layer in DNGSOSCC pattern accepts number of sensor nodes as input. Subsequently, DNGSOSCC model employed four hidden layers with objective of increasing route discovery and data collection performance through accomplishing soft clustering, initial population of glow worms, luciferin value computation and optimal cluster head $(\mathrm{CH})$ selection. After that, the output layer in DNGSOSCC model generates optimal cluster head selection. Finally, chosen cluster head amasses the packets from its member and consequently broadcast the gathered data to sink node with minutest amount of energy absorption. Hence, DNGSOSCC model obtains energy effectual data assortment in WSN.

Fig. 2 illustrates the structural diagram of DNGSOSCC model to get global optimal solution for cluster head selection in sensor networks. As presented in the diagram input, hidden and output layer in DNGSOSCC model are connected to each other. The initial layer comprises input neurons that acquires sensor nodes as input and forward it to the subsequent layer recognised as the hidden layer.

In DNGSOSCC model, four hidden layers are utilised where soft clustering, initial inhabitants of glow worms, luciferin value measurement and

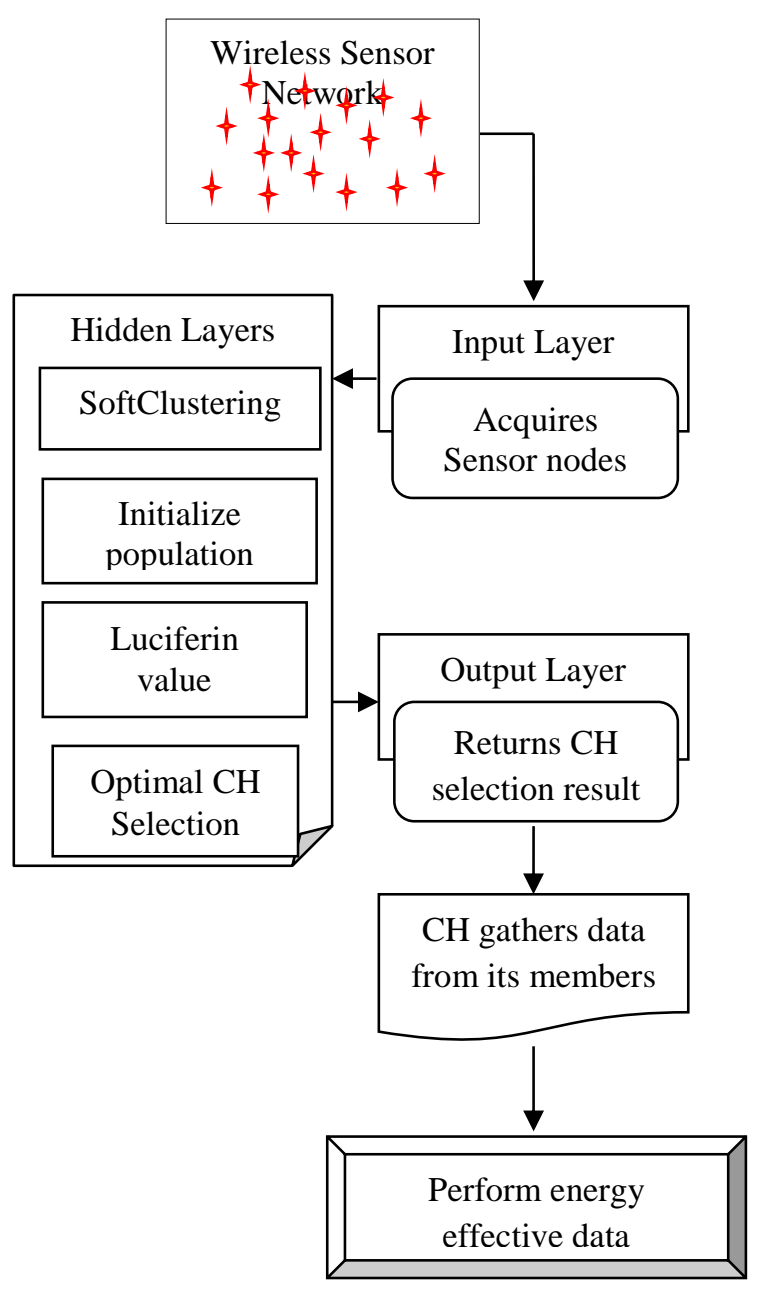

Figure. 1 Architecture diagram of DNGSOSCC model

optimal $\mathrm{CH}$ selection process is accomplished with aiming at reducing the energy utilization during the process of data gathering in WSN. At last, the output layer gives the optimal $\mathrm{CH}$ selection result.

Let us consider WSN comprising huge number of sensor nodes represented as ' $\delta_{1}, \delta_{2}, \delta_{3} \ldots, \delta_{z}$ '. Here, ' $z$ ' denotes the total quantity of sensor nodes in a sensor network. At the beginning, DNGSOSCC model initializes the neural network with arbitrary weights.

The DNGSOSCC model acquires sensor nodes ' $\delta_{i}$ ' as input.After getting the input, the input layer in DNGSOSCC model unite the sensor nodes ' $\delta_{i}$ ' with weights ' $k_{U V}$ ' and bias term ' $a_{j}$ '. Henceforth, input layer ' $(U)$ ' activity is expressed as,

$$
U(t)=\sum \delta_{i} k_{U V}+a_{j}
$$

From Eq. (1), ' $U(t)$ ' refers input layer process at time ' $t$ '. Here, ' $\delta_{i}$ ' signifies an input sensor node and ' $k_{U V}$ ' symbolizes the weight between the input and hidden layer and ' $a_{j}$ ' is a bias. Following the input, the input layer promotes input sensor nodes to hidden 


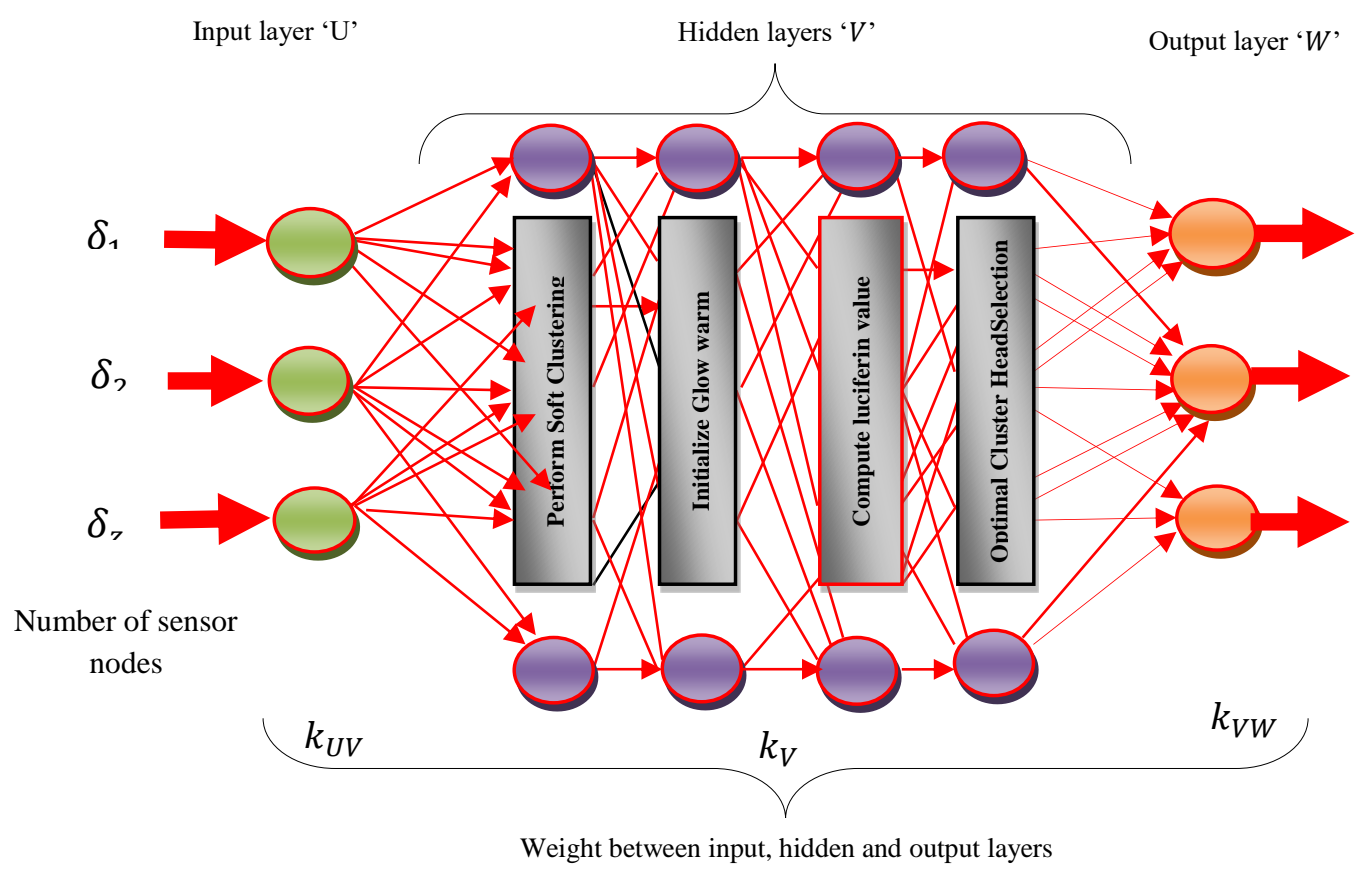

Figure. 2 Structural diagram of DNGSOSCC model

layers. In DNGSOSCC model, first hidden layer performs soft c-means clustering which is described mathematically as,

$$
V_{1}(t)=\sum U(t) k_{V_{1}}[S C C]
$$

From Eq. (2), ' $V_{1}(t)$ ' represents the primary hidden layer process at time ' $t$ ' however ' $U(t)$ ' designates the outputfrom the input layer (i.e. number of nodes) and ' $k_{V_{1}}$ ' signifies weight of initial hidden layer. Here, 'SCC' signifies the soft c-means clustering. During the node grouping process, DNGSOSCC model initialize ' $c$ ' number of clusters ' $c_{1}, c_{2}, c_{3}, \ldots c_{c}$ ' and their cluster centroid ' $b_{1}, b_{2}, b_{3}, \ldots b_{c}$ ' randomly. The soft c-means clustering process is performed by means of assigning membership to each sensor node ' $\delta_{i}$ ', corresponding to each cluster centroid based on distance between the centroid and the node. From that, membership to which a sensor node ' $\delta_{i}$ ' belongs to cluster ' $c_{j}$ ' is obtained as,

$$
M_{i j}=\sum_{n=1}^{c}\left(\frac{\alpha_{i j}}{\alpha_{i c}}\right)^{-\left(\frac{2-f}{f}\right)}
$$

From Eq. (3), ' $M_{i j}$ ' represents a membership function and ' $\alpha_{i j}$ ' indicates a distance between ' $i$ ' , sensor node and their ' $j$ th' cluster centroid. Here, ' $\alpha_{i c}$ ' is a distance between ' $i^{t h}$, sensor node and ' $c^{t h}$, cluster and ' $f$ ' is fuzzifier which is employed to find out the level of cluster fuzziness. The DNGSOSCC Model calculates the cluster centroid as mean of all sensor nodes weighted by their membership degree belongs to the cluster. Accordingly, the centroid for each cluster is evaluated as,

$$
b_{\mathrm{j}}=\frac{\sum \delta_{i} \in c_{j}}{M_{i j}^{f} \delta_{i}}
$$

From Eq. (4), ' $b_{\mathrm{j}}$ ' signifies centroid of the cluster and ' $M_{i j}$ ' is amembership degree. Subsequently, the distance between the node and cluster centroid is estimated as,

$$
\alpha_{i j}=\left(\sum_{i=1}^{z}\left(\left|\left(\delta_{i}-b_{\mathrm{j}}\right)\right|\right)^{q}\right)^{1 / q}
$$

From Eq. (5), ' $b_{\mathrm{j}}$ ' represents a ' $j$ th' cluster centroid and ' $\delta_{i}$ ' represents the ' $i$ 'th' sensor node in network. Here, ' $z$ ' denotes total quantity of sensor node and ' $q$ ' is a parameter. From that, the minimal distance between sensor node and cluster centroid is more suitable to group the sensor node to that particular cluster. By using the above Eqs (3) - (5), first hidden layer efficiently creates the different number of clusters in WSN using soft c-means clustering. The node clustering outcome is then remitted to second hidden layer. During this course, second hidden layer initialize the glow worm population by considering number of clusters formed in WSN. Consequently, second hidden layer process ' $V_{2}(t)$ ' is acquired as,

$$
V_{2}(t)=\sum V_{1}(t) k_{V_{2}}[I P G W]
$$


From Eq. (6), ' $V_{2}(t)$ ' refers second hidden layer result at time ' $t$ ' whereas ' $V_{1}(t)$ ' designates an output from primary hidden layer (i.e. number of node clusters) and ' $k_{V_{2}}$ ' indicates weight of second hidden layer. Here, ' $I P G W$ ' exemplifies the early inhabitants of glow worms with number of clusters formed in WSN which is mathematically expressed as,

$$
I P G W=c_{1}, c_{2}, \ldots c_{c}
$$

From Eq. (7), ' $c$ ' designates a total number of clusters formed in WSN. Once concluding initialization process, DNGSOSCC model accomplishes the luciferin value for every sensor node in clusters at the third hidden layer. Thus, third hidden layer process ' $V_{3}(t)$ ' at a time ' $t$ ' is defined as,

$$
V_{3}(t)=\sum V_{2}(t) k_{V_{3}}, L_{\delta_{i}}
$$

From Eq. (8), ' $V_{3}(t)$ ' indicate third hidden layer result and ' $V_{2}(t)$ ' symbolises the output attained from the second hidden layer i.e. primary inhabitants of glow worms amid number of clusters. Here ' $k_{V_{3}}$ ' represents weight of third hidden layers and ' $L_{\delta_{i}}$ ', indicates the luciferin value of every sensor node. From that, luciferin value for all the sensor nodes in WSN is mathematically calculated as,

$$
L_{\delta_{i}}=R E L
$$

From Eq. (9), luciferin value of each node ' $L_{\delta_{i}}$ ' is measured by considering their residual energy level '( $R E L)$ '. Accordingly, residual energy level is dogged as dissimilarity between the total energy ' $T E$ ' and the energy used ' $E U$ ' using below,

$$
R E L=T E-E U
$$

From Eq. (10), the remaining energy level for entire node is computed. The energy level is approximated by means of joule (J). Subsequently, best $\mathrm{CH}$ is selected for each cluster at the fourth hidden layer based on the behaviour of glow worms (i.e. fireflies). The glow worm with lesser lighting behaviour is magnetized to the brighter one. This helps for fourth hidden layer in DNGSOSCC model to discover the sensor node with maximum luciferin value within cluster as optimal $\mathrm{CH}$. As a result, fourth hidden layer ' $V_{4}(t)$ ' process is explained as,

\begin{tabular}{|c|c|}
\hline Notation & Description \\
\hline$Z$ & Number of nodes \\
\hline$\delta_{i}$ & Sensor nodes \\
\hline$c_{i}$ & Clusters \\
\hline$b_{i}$ & Cluster centroid \\
\hline $\mathrm{F}$ & Fuzzifier \\
\hline$q$ & parameter \\
\hline$a_{j}$ & Bias term \\
\hline$k_{u v}$ & $\begin{array}{l}\text { Weights between input layer } \\
\text { and hidden layer }\end{array}$ \\
\hline$k_{v}$ & Weights of hidden layer \\
\hline$k_{v w}$ & $\begin{array}{l}\text { Weights between hidden and } \\
\text { output layer }\end{array}$ \\
\hline$U(t)$ & Input layer at time ' $\mathrm{t}$ ' \\
\hline$V(t)$ & Hidden layer result at time ' $t$ ' \\
\hline$M_{i j}$ & Membership function \\
\hline$I P G W$ & $\begin{array}{l}\text { Initial population of glow } \\
\text { worm }\end{array}$ \\
\hline$L_{\delta_{i}}$ & Luciferin value of sensor node \\
\hline$R E L$ & Residual energy \\
\hline$T E$ & Total Energy \\
\hline$E U$ & Energy Used \\
\hline$S C C$ & Soft $\mathrm{C}$ means clustering \\
\hline$A$ & Activation Function \\
\hline$e(t)$ & Error rate \\
\hline$\overline{\overline{W(t)}}$ & Target output \\
\hline$W(t)$ & Actual output \\
\hline$D D R$ & Data deliverance Rate \\
\hline$D L R$ & Data Loss Rate \\
\hline DTD & Data transmission delay \\
\hline$D S T$ & Data Sent time \\
\hline$N L$ & Network Lifetime \\
\hline
\end{tabular}

$$
\begin{aligned}
& V_{4}(t)=\sum V_{3}(t) k_{V_{4}} \\
& {\left[\text { optimalCH } \leftarrow \arg \max \left(L_{\delta_{i}} \in c_{i}\right)\right]}
\end{aligned}
$$

Table 1. Tabulation for list of notations

From Eq. (11), ' $V_{4}(t)$ ' reveals the fourth hidden layer outcome at time ' $t$ ' and ' $V_{3}(t)$ ' is the output taken from the third hidden layer (i.e. luciferin value of nodes) and ' $k_{V_{4}}$ ' serves weight of fourth hidden layers. Here, 'optimal $C H \leftarrow \arg \max \left(L_{\delta_{i}} \in c_{i}\right)$ ' denotes optimal $\mathrm{CH}$ chosen process within cluster based on higher luciferin value. The optimal $\mathrm{CH}$ selected result for each cluster in WSN is subsequently directed to output layer. The output layer ' $W(t)$ ' process at time ' $t$ ' is defined as,

$$
W(t)=A k_{V W} V_{4}(t)
$$

From Eq. (12), ' $k_{V W}$ ' implies weight between hidden and output layer and ' $A$ ' is an activation function. The output layer in DNGSOSCC model produces optimal $\mathrm{CH}$ selected for each cluster in WSN. For each obtained result at output layer, the DNGSOSCC model measures error rate ' $e(t)$ ' using below, 
The algorithmic processes of DNGSOSCC model is explained in below,

//Deep Neural Glow worm Swarm Optimized // Soft C-Means Clustering Algorithm

Input: Number of sensor nodes ' $\delta_{1}, \delta_{2}, \delta_{3}, \ldots, \delta_{n}$,' Output: Obtain the energy aware route discovery and data gathering In WSN

\section{Step 1: Begin}

Step 2: Define artificial neural network with random weights

Step 3: While (network error is very lower) do

Step 4: For number of sensor nodes in WSN

// Input layer

Step 5: Takes nodes as input

// First hidden layer

Step 6: Carry out soft c-means clustering process // Second hidden layer

Step 7: Initialize glow worm population with number of clusters in WSN

// Third hidden layer

Step 8: $\quad$ Calculate luciferin value // Fourth hidden layer

Step 9: Choose node with higher luciferin value within a cluster as optimal cluster head

// Output layer

Step 10: Returns optimal cluster head selection

Step 11: End For

Step 12: $\quad$ For each result at output layer

Step 13: Determine error

Step 14: Update weights on network

Step 15: End For

Step 16: End While

Step 17: For each cluster in WSN

Step 18: Cluster head gathers data from its members

Step 19: Cluster head sent collected data to sink

Step 20: Achieve energy efficient data gathering in WSN

\section{Step 21: End For}

Step 22: End

Algorithm 1 Deep neural glow-worm swarm optimized soft c-means clustering

DNGSOSCC model measures error rate ' $e(t)$ ' using below,

$$
e(t)=\overline{W(t)}-W(t)
$$

From Eq. (13), DNGSOSCC model estimates error rate as differentiation between a target output ' $\overline{W(t)}$ ' and actual output ' $W(t)$. Subsequently, DNGSOSCC model updates the weights on network based on calculated error rate. The above processes of DNGSOSCC model is continual until the error value is very minimum to accurately find best $\mathrm{CH}$ for each cluster constructed in WSN. After completing $\mathrm{CH}$ selection process, DNGSOSCC model collects data from cluster member with help of selected $\mathrm{CH}$ and finally sent the gathered data to sink node with lower amount of energy.

Algorithm 1 describes the steps of DNGSOSCC model to reduce the energy usage of data collection in WSN via optimal cluster head selection. DNGSOSCC model at first initialize the artificial neural network with arbitrary weights. Followed by, DNGSOSCC model acquires number of sensor nodes in wireless network as input at input layer. After getting the input, DNGSOSCC model groups the similar types of sensor nodes together at the first hidden layer with help of soft c-means clustering process. In the second hidden layer, subsequently DNGSOSCC model initialize the glow worm swarm population by considering number of node clusters in WSN. Then, DNGSOSCC model determines the Luciferin value in the third hidden layer for all the sensor nodes in each cluster implanted on their enduring energy level. In the fourth hidden layer, DNGSOSCC model finds sensor node with higher Luciferin value as optimal cluster head for each cluster in WSN to carryout data aggregation in WSN with minimal energy. After that, output layer returns the optimal cluster head selection.

For each acquired result at output layer, the DNGSOSCC model computes error rate and consequently updates weights on neural network. The process of DNGSOSCC model is repeated until the neural network error is very lower for optimal selection of cluster head in WSN. Finally, DNGSOSCC model aggregates the data from its members for each cluster in WSN through an optimal cluster head with lower energy. This helps for DNGSOSCC model to provide improved route discovery and data gathering accomplishment in requisites of data deliverance rate, data loss rate, and data dispersion delay and network lifetime in WSN while correlated to conventional works.

\section{Simulation settings}

The proposed DNGSOSCC replica is materialized in NS-2 simulator by examining wireless network region of $1500 \mathrm{~m} * 1500 \mathrm{~m}$ using 500 sensor nodes. To perform simulation work, DNGSOSCC model used Random Waypoint model as mobility and DSR as routing protocol. The simulation parameter utilized for conducing experimental process is depicted in Table 2 . 
Table 2. Simulacrum features

\begin{tabular}{|c|c|}
\hline Simulacrum Features & Assesses \\
\hline Network Simulator & NS 2.34 \\
\hline Square space & $1500 \mathrm{~m} \times 1500 \mathrm{~m}$ \\
\hline $\begin{array}{c}\text { Number of sensor } \\
\text { nodes }\end{array}$ & 500 \\
\hline Mobility standard & $\begin{array}{l}\text { Random Waypoint } \\
\text { model }\end{array}$ \\
\hline Speed of sensor nodes & $0-20 \mathrm{~m} / \mathrm{s}$ \\
\hline Counterfeit time & $250 \mathrm{sec}$ \\
\hline Protocol & DSR \\
\hline Number of runs & 10 \\
\hline
\end{tabular}

The simulation measure of DNGSOSCC pattern is determined in stipulations of data deliverance rate, data loss rate and data transmission delay and network lifetime. The simulation result of DNGSOSCC model is compared against traditional fuzzy based balanced cost $\mathrm{CH}$ selection algorithm (FBECS) [1] and enhanced clustering hierarchy (ECH) [2] approach.

\section{Results and discussion}

The simulation performance of DNGSOSCC model is analysed and compared with two existing methods namely FBECS [1] and ECH [2] approach.

\subsection{Data deliverance rate}

Data Deliverance Rate ' $D D R$ ' determines proportionate of total data successfully reached at target to the total data disseminated by source node. The data delivery rate is obtained as,

$$
D D R=\left(\begin{array}{c}
\text { Number of data } \\
\text { successfully reached }
\end{array}\right) \times 100
$$

From Eq. (14), ' $n$ ' refers a total data packets propagated. The data deliverance rate is measured in terms of percent $(\%)$. The experimental result of data liberation rate is determined for the period of data collection in WSN using proposed DNGSOSCC model and conventional FBECS [1] and ECH [2] along with various number of data packets shown in below Table 3 .

Table 3 presents the gust of data delivery rate in WSN using proposed DNGSOSCC model and conventional FBECS [1] and ECH [2] with reverence to assorted number of packets in the scope of 25-250. Let us consider a simulation environment of $1500 \mathrm{~m}$ * 1500 m wireless network area with 225 collected data packets. During the simulacrum process,
Table 3. Outcome for data deliverance rate

\begin{tabular}{|l|l|l|l|}
\hline \multirow{2}{*}{$\begin{array}{l}\text { Total } \\
\text { Data } \\
\text { packets }\end{array}$} & \multicolumn{4}{|l|}{ Data deliverance ratio (\%) } \\
\cline { 2 - 4 } & DNGSOSCC & FBECS & ECH \\
\hline 25 & 96 & 80 & 72 \\
\hline 50 & 94 & 82 & 76 \\
\hline 75 & 97 & 81 & 77 \\
\hline 100 & 95 & 85 & 81 \\
\hline 125 & 95 & 81 & 79 \\
\hline 150 & 93 & 83 & 78 \\
\hline 175 & 95 & 81 & 79 \\
\hline 200 & 94 & 83 & 81 \\
\hline 225 & 98 & 80 & 78 \\
\hline 250 & 96 & 83 & 80 \\
\hline
\end{tabular}

projected DNGSOSCC replica achieves $98 \%$ data delivery rate whereas traditional FBECS [1] and ECH [2] gets $80 \%$ and $78 \%$ respectively. As depicted in the above graphical result analysis, it is considerable that the data delivery date using proposed DNGSOSCC model is high when correlated to previous works [1] and [2]. This is due to appliance of glow worm swarm optimization and soft c-means clustering and deep neural learning concepts in planned DNGSOSCC model. Divergent to the existing mechanisms, proposed DNGSOSCC model enhances optimal cluster head selection performance in WSN. This helps proposed DNGSOSCC pattern to choose the higher residual energy sensor nodes as best cluster head amidst the data gathering procedure. With assist of cluster head with higher residual energy, proposed DNGSOSCC model increases the stability of routing the packets from source to end nodes in WSN. As a result, percentage of total packets successfully reached at the target end using proposed DNGSOSCC model is improved when compared to existing FBECS [1] and ECH [2].

\subsection{Data loss rate}

Data loss rate evaluates proportion of total data packet expelled during the data aggregation process to the total packets sent by source node. The data loss rate is computed using the beneath expression,

$$
D L R=\left(\frac{\begin{array}{l}
\text { Number of data } \\
\text { packets dropped }
\end{array}}{n}\right) \times 100
$$

From Eq. (15), ' $n$ ' indicate a total packets broadcasted by source node. The data loss rate is determined in the terms of proportion (\%). The accomplishment assess of data loss rate is evaluated during the process of data gathering in WSN using proposed DNGSOSCC model and traditional FBECS [1] and ECH [2] built on dissimilar quantity of 
Table 4. Outcome for data loss rate

\begin{tabular}{|l|l|l|l|}
\hline \multirow{2}{*}{$\begin{array}{l}\text { Total } \\
\text { Data } \\
\text { packets }\end{array}$} & \multicolumn{4}{|l|}{ Data loss ratio $(\%)$} \\
\cline { 2 - 4 } & DNGSOSCC & FBECS & ECH \\
\hline 25 & 4 & 20 & 28 \\
\hline 50 & 6 & 18 & 24 \\
\hline 75 & 3 & 19 & 23 \\
\hline 100 & 5 & 15 & 19 \\
\hline 125 & 5 & 19 & 21 \\
\hline 150 & 7 & 17 & 22 \\
\hline 175 & 5 & 19 & 21 \\
\hline 200 & 6 & 18 & 20 \\
\hline 225 & 2 & 20 & 22 \\
\hline 250 & 4 & 17 & 20 \\
\hline
\end{tabular}

data packets and illustrated in Table 4 .

Table 4 shows the statistical result measurement of data loss rate in WSN using proposed DNGSOSCC model and traditional FBECS [1] and $\mathrm{ECH}$ [2] along with diverse number of packets in the scope of 25-250. While conducting simulation process, proposed DNGSOSCC model averagely minimizes data loss rate by $74 \%$ whilst compared to contemporary FBECS [1] and 78\% when compared to existing ECH [2]. This is owing to employment of glow worm swarm optimization, soft c-means clustering and deep neural learning concepts in proposed DNGSOSCC model. During the data aggregation process, proposed DNGSOSCC model decreases the data loss rate by choosing the cluster head with maximum residual energy as best among the number of sensor nodes in every cluster. This supports for proposed DNGSOSCC model to avoid the information loss due to path failure and energy limitations. As a result, proposed DNGSOSCC model reduces number of data packet dropped during the data aggregation course in WSN whilst compared to conventional FBECS [1] and ECH [2].

\subsection{Data transmission delay}

Data Transmission Delay (DTD) estimated as the dissimilarity among the transmitting and acceptance period of data in WSN. The data transmittal delay is determined as,

$$
D T D=(D R T-D S T)
$$

From Eq. (16), ' $D R T$ ' exemplifies data reception time, ' $D S T$ ' refers a data sent time. The delay is evaluated in milliseconds (ms). The simulacrum consequence of data transmittal delay is measured amidst the data aggregation course in WSN using
Table 5. Outcome for data transmission delay

\begin{tabular}{|l|l|l|l|}
\hline \multirow{2}{*}{$\begin{array}{l}\text { Total } \\
\text { Data } \\
\text { packets }\end{array}$} & \multicolumn{3}{|l|}{ Data Transmission Delay (ms) } \\
\cline { 2 - 4 } & DNGSOSCC & FBECS & ECH \\
\hline 25 & 8 & 16 & 20 \\
\hline 50 & 10 & 19 & 22 \\
\hline 75 & 13 & 24 & 29 \\
\hline 100 & 19 & 27 & 30 \\
\hline 125 & 21 & 28 & 33 \\
\hline 150 & 24 & 30 & 35 \\
\hline 175 & 25 & 33 & 36 \\
\hline 200 & 29 & 36 & 40 \\
\hline 225 & 32 & 39 & 43 \\
\hline 250 & 33 & 41 & 47 \\
\hline
\end{tabular}

proposed DNGSOSCC model and existing FBECS [1] and ECH [2] with deference to varied number of packets and shown in Table 5.

Table 5 illustrates the performance result estimation of data transmission delay for the duration of aggregation course in WSN using proposed DNGSOSCC model and conventional FBECS [1] and $\mathrm{ECH}$ [2] according to diverse number of packets in the sequence of 25-250. The proposed DNGSOSCC model gets $24 \mathrm{~ms}$ data transmission delay when performing an experimental evaluation with 150 data packets whereas state-of-the-art FBECS [1] and ECH [2] obtains 30ms and 35ms respectively. Thus, it is clear that the transmission delay involved during the data collection process in WSN using proposed DNGSOSCC model is lesser when related to previous mechanisms. This is owing to process of glow worm swarm optimization and soft c-means clustering and deep neural learning concepts in proposed DNGSOSCC model. Hence, proposed DNGSOSCC model reduces the variation amid the transmitting and acceptance period of data in WSN whilst correlated to current FBECS [1] and ECH [2].

\subsection{Network lifetime}

Network Lifetime ' $N L$ ' is measured as the proportion of a number of privileged energy nodes elected for routing data packets to destination to the total quantity of nodes in WSN. The network life span is computed as follows,

$$
\mathrm{NL}=\left(\frac{\begin{array}{c}
\text { Number of higher energy } \\
\text { nodes are selected }
\end{array}}{n}\right) \times 100
$$

From Eq. (17), ' $n$ ' denotes overall number of sensor nodes. The network duration is evaluated in percentage $(\%)$. The simulation result of network lifetime is measured amid the data aggregate process 
Table 6. Outcome of network lifetime

\begin{tabular}{|l|l|l|l|}
\hline \multirow{2}{*}{$\begin{array}{l}\text { Total } \\
\text { sensor } \\
\text { nodes }\end{array}$} & Network Lifetime (\%) \\
\cline { 2 - 4 } & DNGSOSCC & FBECS & ECH \\
\hline 50 & 92 & 82 & 78 \\
\hline 100 & 95 & 86 & 83 \\
\hline 150 & 93 & 88 & 86 \\
\hline 200 & 97 & 89 & 86 \\
\hline 250 & 95 & 87 & 85 \\
\hline 300 & 93 & 90 & 87 \\
\hline 350 & 97 & 91 & 89 \\
\hline 400 & 98 & 88 & 85 \\
\hline 450 & 97 & 89 & 87 \\
\hline 500 & 95 & 87 & 85 \\
\hline
\end{tabular}

in WSN using proposed DNGSOSCC model and existing FBECS [1] and ECH [2] with revere to varied number of packets and shown in Table 6 .

Table 6 illuminates the comparative result evaluation of network lifetime when performing energy aware route discovery and data aggregation process in WSN using three process based on dissimilar total sensor nodes in the series of 50-500. When accomplishing simulation work by taking 200 nodes in wireless network, proposed DNGSOSCC pattern averagely enhances network life span by $9 \%$ while compared to conventional FBECS [1] and $12 \%$ when compared to traditional ECH [2]. This is due to usage of glow worm swarm optimization and soft cmeans clustering and deep neural learning concepts in proposed DNGSOSCC model. During the implementation of proposed DNGSOSCC model, nodes which contain a privileged lingering energy level are chosen as best cluster head to gather data packets from cluster members on the converse to conservative works. Thus, proposed DNGSOSCC technique increases the network lifetime when compared to traditional FBECS [1] and ECH [2].

\section{Conclusion}

In this manuscript, an effective DNGSOSCC model is recommended with the aim of achieving energy proficient data aggregation in WSN by optimal cluster head selection. The aim of DNGSOSCC model is obtained with the application of glow worm swarm optimization, soft c-means clustering and deep neural learning concepts. Based on these concepts, proposed DNGSOSCC model gets enhanced cluster head selection performance with a minimal chunk of time when correlated to existing mechanisms. Also, proposed DNGSOSCC sculpt decreases the vitality usage during the data collection process by finding the optimal cluster heads with maximum residual energy when correlated to conventional mechanisms. Moreover, the planned DNGSOSCC technique increases the network lifetime when compared to the conventional works. The recommended DNGSOSCC technique obtains enhanced routing achievement in terms of data deliverance ratio, data loss rate and data transmission delay through discovering an optimal route path between source and destination with higher stability. The simulation result depicts that proposed DNGSOSCC model improves the data deliverance ratio and network lifetime by $19 \%$ and $10 \%$ as well as minimizes the data loss ratio and delay by $76 \%$ and $34 \%$ respectively than existing methods. In future, data transmission delay can be reduced and network lifetime can be improved by using ensemble classification with optimization techniques for performing route path discovery and data gathering in WSN.

\section{Conflicts of Interest}

The authors declare no conflict of interest.

\section{Author Contributions}

Conceptualization, Sridhar Ramaiah and Guruprasad Nagraj; methodology, Sridhar Ramaiah and Guruprasad Nagraj; Software, Sridhar Ramaiah; formal analysis, Sridhar Ramaiah and Guruprasad Nagraj; investigation, Sridhar Ramaiah and Guruprasad Nagraj; writing-original draft preparation, Sridhar Ramaiah; writing-review and editing, Guruprasad Nagraj; supervision, Guruprasad Nagraj: project administration, Sridhar Ramaiah and Guruprasad Nagraj.

\section{References}

[1] P. S. Mehra, M. Najmud, and D. B. Alam, "Fuzzy based enhanced cluster head selection (FBECS) for WSN", Journal of King Saud University - Science, Elsevier, Vol. 32, No. 1, pp. 390-401, 2020.

[2] H. E. Alami and A. Najid, "ECH: An Enhanced Clustering Hierarchy Approach to Maximize Lifetime of Wireless Sensor Networks", IEEE Access, Vol. 7, pp. 107142 - 107153, 2019.

[3] D. Takaishi, H. Nishiyama, N. Kato and R. Miura, "Toward Energy Efficient Big Data Gathering in Densely Distributed Sensor Networks", IEEE Transactions on Emerging Topics in Computing, Vol. 2, No. 3, pp. $388-$ 397, 2014.

[4] B. Rambabu, A. V. Reddy, and S. Janakiraman, "Hybrid Artificial Bee Colony and Monarchy 
Butterfly Optimization Algorithm (HABCMBOA)-based cluster head selection for WSNs", Journal of King Saud University - Computer and Information Sciences, Elsevier, pp. 1-11, 2019.

[5] A. Sarkar and T. S. Murugan, "Cluster head selection for energy efficient and delay-less routing in wireless sensor network", Wireless Networks, Springer, Vol. 25, pp. 303-320, 2019.

[6] D. Ebrahimi, S. Sharafeddine, P. H. Ho, and C. Assi, "UAV-Aided Projection-Based Compressive Data Gathering in Wireless Sensor Networks", IEEE Internet of Things Journal, Vol. 6, No. 2, pp.1893 - 1905, 2019.

[7] R. Sharma, V. Vashisht, and U. Singh, "Fuzzy modelling-based energy aware clustering in wireless sensor networks using modified invasive weed optimization", Journal of King Saud University - Computer and Information Sciences, Elsevier, pp. 1-11, 2019.

[8] S. Famila and A. Jawahar, "Improved Artificial Bee Colony Optimization-Based Clustering Technique for WSNs", Wireless Personal Communications, Springer, Vol. 110, pp. 21952212, 2020.

[9] Y. Hu, Y. Niu, J. Lam, and Zhan Shu, "An Energy-Efficient Adaptive Overlapping Clustering Method for Dynamic Continuous Monitoring in WSNs", IEEE Sensors Journal, Vol. 17, No. 3, pp. 834 - 847, 2017.

[10] K. N. Dattatraya and K. R. Rao, "Hybrid based cluster head selection for maximizing network lifetime and energy efficiency in WSN", Journal of King Saud University - Computer and Information Sciences, Elsevier, pp. 1-11, 2019.

[11] R. Sharma, V. Vashisht, and U. Singh, "eeTMFO/GA: a secure and energy efficient cluster head selection in wireless sensor networks", Telecommunication Systems, Vol. 21, pp. 1-16, 2020.

[12] S. Verma, N. Sood, and A. K. Sharma, "Genetic Algorithm-based Optimized Cluster Head selection for single and multiple data sinks in Heterogeneous Wireless Sensor Network", Applied Soft Computing, Elsevier, Vol. 85, pp. 1-21, 2019.

[13] G. Li, B. He, Z. Wang, S. Xu, and H. Huang, "A Swarm Optimization-Enhanced Data Aggregation Tree Based on a Nonuniform Clustering Structure for Long and Linear Wireless Sensor Networks", Wireless Personal Communications, Springer, 2020.

[14] M. Narendran and P. Prakasam, "An energy aware competition based clustering for cluster head selection in wireless sensor network with mobility", Cluster Computing, Springer, Vol. 22, pp. 11019-11028, 2019.

[15] R. Hamidouche, Z. Aliouat, A. A. A. Ari, and M. Gueroui, "An Efficient Clustering Strategy Avoiding Buffer Overflow in IoT Sensors: A Bio-Inspired Based Approach", IEEE Access, Vol. 7, pp. 156733 - 156751, 2019.

[16] P. K. Poonguzhali and N. P. Ananthamoorthy, "Improved energy efficient WSN using ACO based HSA for optimal cluster head selection", Peer-to-Peer Networking and Applications, Springer, pp. 1-7, 2019.

[17] S. Singh, "A sustainable data gathering technique based on nature inspired optimization in WSNs", Sustainable Computing: Informatics and Systems, Elsevier, Vol. 24, pp. 1-35, 2019.

[18] A. Shankar, N. Jaisankar, M. S. Khan, R. Patan, and B. Balamurugan, "Hybrid model for security-aware cluster head selection in wireless sensor networks", IET Wireless Sensor Systems, Vol. 9, No. 2, pp. 68 - 76, 2019.

[19] A. A. Baradaran and K. Navi, "HQCA-WSN: High-quality clustering algorithm and optimal cluster head selection using fuzzy logic in wireless sensor networks", Fuzzy Sets and Systems, Elsevier, Vol. 389, pp. 114-144, 2020.

[20] A. P. Jyothi and S. Usha, "MSoC: Multi-scale Optimized Clustering for Energy Preservation in Wireless Sensor Network", Wireless Personal Communications, Vol. 105, pp. 1309-1328, 2019. 